\title{
Potentialités agroforestières de Maranthes polyandra (Benth.) Prance au sud-ouest du Burkina Faso
}

\author{
* Paulin Ouoba1 ${ }^{3}$, Jérôme T.Yaméogo², Amadé Ouédraogo 3 , Souleymane Kouaman² \\ 1 Université Nazi Boni, Unité de formation et de recherche en sciences et techniques (UFR-ST), Département de Sciences \\ biologiques. 01 BP 1091 Bobo-Dioulasso 01, Burkina Faso \\ 2Université Nazi Boni, institut du développement rural (IDR), Département des Eaux et Forêts. 01 BP 1091 Bobo- \\ Dioulasso 01, Burkina Faso \\ 3Université Ouaga1 Joseph Ki-Zerbo, Unité de formation et de recherche en sciences de la Vie et de la Terre (UFR-SVT), \\ Laboratoire de Biologie et Écologie Végétale (LABEV). 03 BP 7021 Ouagadougou 01, Burkina Faso \\ *Auteur correspondant : ouobapaulin@hotmail.com
}

Original submitted in on 23 $3^{\text {rd }}$ April 2018. Published online at www.m.elewa.org on $31^{\text {st }}$ August 2018 https://dx.doi.org/10.4314/jab.v128i1.5

\section{RÉSUMÉ}

Objectif : Le maintien d'arbres et d'arbustes dans les champs, en association avec les cultures est certes lié à leur utilité alimentaire, médicinale, mais aussi à leurs aptitudes à améliorer la fertilité des sols. Des observations ont révélé une flore ligneuse abondante qui pousse préférentiellement au pied de Maranthes polyandra (Benth.) Prance. Elles ont suscité l'hypothèse que $M$. polyandra a des propriétés agro-écologiques intéressantes. L'objectif de l'étude est d'évaluer les relations entre $M$. polyandra et les propriétés physiques et chimiques du sol.

Méthodes et résultats : La quantité de litière foliaire sous le houppier de $M$. polyandra a été collectée et pesée. Des échantillons de sol ont été prélevés sous le houppier et hors du houppier de M. polyandra pour des analyses physico-chimiques. Les mêmes travaux ont été réalisés à titre comparatif avec Vitellaria paradoxa C.F.Gaertn., qui est une espèce agroforestière connue.

La quantité moyenne de litière foliaire sous $M$. polyandra est de $28,82 \pm 16,08 \mathrm{~kg}$ par individu contre $5,92 \pm$ $3,07 \mathrm{~kg}$ pour $\mathrm{V}$. paradoxa. Nous avons trouvé des teneurs significativement plus élevées en carbone total, en azote total, en phosphore total, en potassium total et en somme des bases échangeables sous M. polyandra, comparativement à $V$. paradoxa. Les paramètres de texture sont significativement meilleurs pour le sol sous houppier de M. polyandra.

Conclusion et application des résultats : Ce travail à permis d'étudier les relations entre $M$. polyandra et les propriétés physiques et chimiques du sol. Il a montré que l'espèce a un potentiel à améliorer la fertilité physique et chimique du sol. Maranthes polyandra pourrait être adoptée comme espèce agroforestière pour améliorer la fertilité du sol. Au Burkina Faso, plus de la moitié des terres sont pauvres en matière organique, en phosphore et en azote. Ces niveaux bas constituent la contrainte majeure pour la production agricole. Ainsi, M. polyandra pourrait être adoptée comme espèce agroforestière pour améliorer la fertilité du sol en plus de son exploitation pour l'alimentation et comme bois d'énergie ; Cela pourrait constituer une raison supplémentaire pour sa conservation.

Mots clés : Agroforesterie, Maranthes polyandra, Fertilité du sol, Burkina Faso, Domestication, Conservation 


\section{ABSTRACT}

\section{Agroforestry potentials of Maranthes polyandra (Benth.) Prance in the south-west of Burkina Faso}

Objective: Maintaining trees and shrubs in fields in association with crops is linked to their use as food, firewood and pharmacopoeia, but also to their ability to improve physicochemical properties of soils. Observations revealed the presence of many plant species that grow preferentially at the foot of Maranthes polyandra (Benth.) Prance. These observations led to the hypothesis that $M$. polyandra has interesting agroecological properties. The objective of the study is to assess the relationship between $M$. polyandra and the physical and chemical properties of soil.

Method and Results. The leaf litter under the crown of M. polyandra was collected and weighed. Soil samples were collected under the crown and outside the crown of $M$. polyandra for physicochemical analyses. The same work was carried out with Vitellaria paradoxa C.F. Gaertn. which is a known agroforestry species.

The litter under M. polyandra is $28.82 \pm 16.08 \mathrm{~kg}$ per individual plant compared to $5.92 \pm 3.07 \mathrm{~kg}$ for $V$. paradoxa. Physicochemical analysis of the soil revealed, higher contents of organic carbon, total nitrogen, total phosphorus, total potassium and values in the sum of exchangeable bases under M. polyandra compared with those obtained under $V$. paradoxa. These texture parameters are significantly better for the soil under crowns of M. polyandra.

Conclusion and application of results: This work studied the relations between M. polyandra and the physical and chemical properties of the soil. It has shown that the species has potential to improve the physical and chemical fertility of the soil. Maranthes polyandra could be adopted as an agroforestry specie to improve soil fertility by its ability to enhance organic matter, phosphorus, nitrogen and potassium of the soils. In Burkina Faso, more than half of the land is poor in organic matter, in phosphorus and in nitrogen. This low levels are the major constraint for agricultural production. Thus, M. polyandra can be used to improve soils fertility in addition to its exploitation as food and firewood. This may be an additional reason for its conservation.

Keywords: Agroforestry, Maranthes polyandra, Soil fertility, Burkina Faso, Domestication, Conservation

\section{INTRODUCTION}

L'utilisation des arbres dans le maintien et la restauration de la fertilité des sols dans les zones arides et semi-arides de l'Afrique de l'Ouest est séculaire (Bationo et Buerkert, 2001). Le paysage agraire dans ces zones est souvent caractérisé par le système de parcs agroforestiers. Le maintien d'arbres et d'arbustes dispersés dans les champs, en association avec les cultures, est certes lié à leurs utilisations dans l'alimentation humaine, le bois de chauffe et de construction, la pharmacopée, le fourrage pour les animaux, mais certains y sont maintenus pour améliorer la fertilité des sols (Bationo et al., 2012). Plusieurs études ont montré l'efficacité de l'agroforesterie dans le maintien et l'amélioration de la fertilité du sol. Elle contribue à la lutte contre l'érosion hydrique et éolienne et à la réduction de l'apport d'engrais minéraux et organiques (Bayala, 2003, Yélémou, 2013; Coulibaly et al., 2016). Aussi, le stockage du carbone, des résidus organiques et la conservation de la biodiversité dans les zones en culture ont été attribués à la présence des ligneux associés (Bayala et al., 2006; Makumba et al., 2007 ; Scherr et Sthapit, 2009). La raréfaction et la dégradation continue des terres cultivables se posent de nos jours avec acuité en Afrique de l'Ouest (Traoré et al., 2007 ; Coulibaly et al., 2012 ; Yaméogo et al., 2013) et la recherche de nouvelles espèces agroforestières éligibles pour la résilience des sols dégradés est un impératif. II a été révélé que plusieurs espèces végétales ligneuses en Afrique de l'Ouest peuvent agir positivement sur leur environnement, surtout dans la fertilisation du sol ; c'est le cas de Guiera senegalensis J.F. Gmel. (Bationo, 1994), de Parkia biglobosa (Jacq.) R. Br. ex G. Don (Bayala et al., 2003) et de Faidherbia albida (Delile) A.Chev. (Roupsard, 1997). Par contre, M. polyandra n'est pas encore connu dans ce domaine. Pourtant les observations montrent la présence de nombreuses espèces végétales qui poussent préférentiellement au pied de cette espèce (Ouoba, 2006). En outre, l'espace sous houppier de $M$. polyandra se 
caractérise par la présence d'une forte quantité de litière constituée de ses feuilles. Ces observations suscitent l'hypothèse que $M$. polyandra a des propriétés agro-forestières intéressantes qui peuvent être valorisées, en plus de son exploitation dans l'alimentation et le bois de chauffe (Arbonnier, 2009; Ouoba et al., 2006). C'est ce qui justifie la présente étude qui a été conduite dans la Forêt Classée de Niangoloko au sud-ouest du Burkina Faso afin d'évaluer les aptitudes de $M$. polyandra dans la fertilisation des sols. Afin de mettre en évidence ces aptitudes, une comparaison est faite avec le karité, V.paradoxa, qui est également fréquente dans la réserve naturelle et classée parmi les espèces

\section{MATÉRIEL ET MÉTHODES}

Zone d'étude et habitat de $M$. polyandra : L'étude a été réalisée dans la Forêt Classée de Niangoloko qui est une réserve naturelle riveraine de la commune de Niangoloko (Figure 1). La zone appartient au climat de type soudanien (Fontes \& Guinko, 1995) et se caractérise par deux (2) saisons contrastées : une saison pluvieuse qui agroforestières les mieux connues de la zone soudanienne de l'Afrique (Weigel, 1994 ; Kaboré et al., 2012). En outre, la problématique de la fertilisation des sols par cette espèce a déjà été abordée par certains auteurs (Diarra, 2009 ; Saidou et al.,2012) au-delà de l'exploitation de ses fruits par I'homme. L'objectif de cette étude était d'étudier les relations entre $M$. polyandra et les propriétés physiques et chimiques du sol. Nous avons supposé que les sols sous houppier de M. polyandra sont plus argileux et ont de meilleures propriétés chimiques que les sols hors houppier de $M$. polyandra.

dure 6 à 8 mois (mars à octobre) au cours de laquelle la pluviométrie moyenne annuelle se situe entre 1000 et $1200 \mathrm{~mm}$ et une saison sèche qui va de novembre à février. Les températures moyennes varient entre 10 et $39^{\circ} \mathrm{C}$, avec un maximum observé entre les mois de mars et avril.

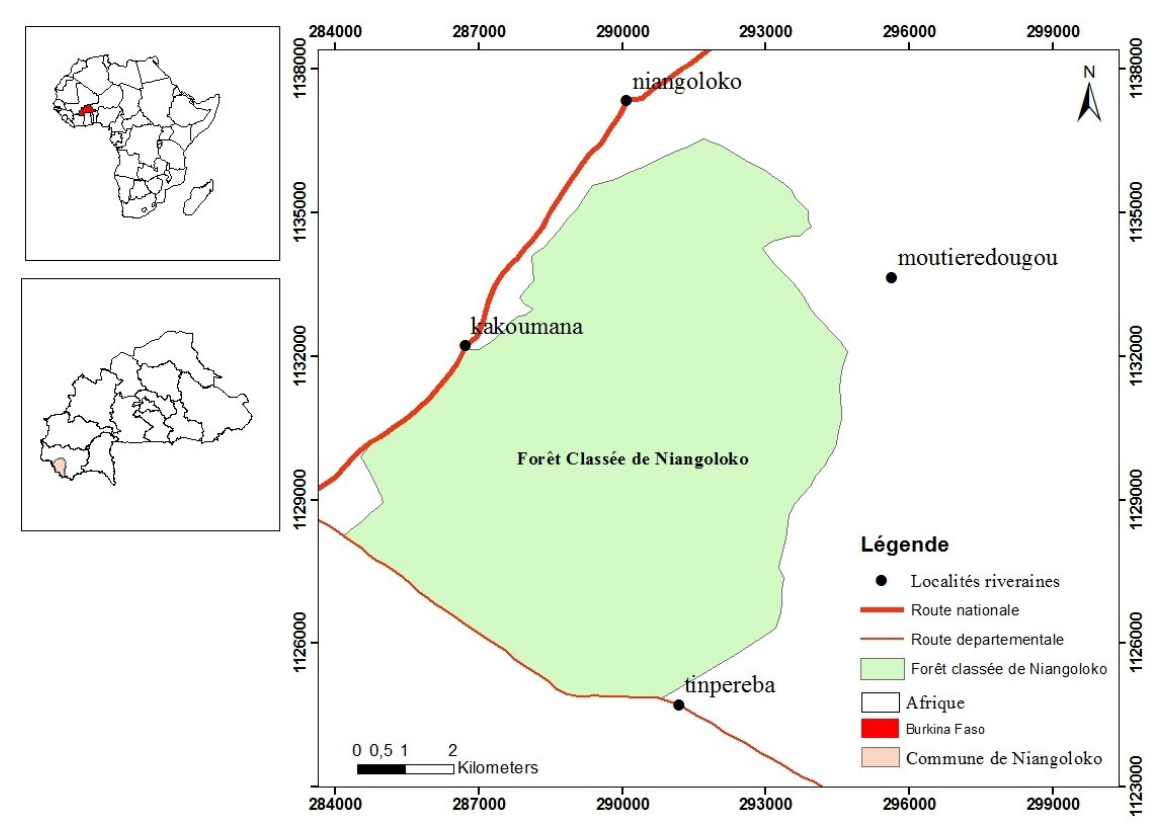

Figure 1 : Localisation de la zone d'étude

Le paysage géomorphologique de la forêt classée est caractérisé par une succession de croupes à sommets convexes ou plats; de ces croupes s'étirent de longs glacis de raccordement dont les pentes varient entre 1 et $3 \%$ (BUNASOL, 1996). En outre, de nombreux escarpements sont observés dans la forêt classée, surtout dans sa portion sud (BUNASOL, 1996). L'altitude varie entre $310 \mathrm{~m}$ et $350 \mathrm{~m}$. Le réseau hydrographique de la forêt classée comprend de nombreux cours d'eau à écoulement temporaire (Ouoba, 2006). Dans cette réserve naturelle, $M$. polyandra fait partie des espèces dominantes de la végétation (Ouoba, 2006). En outre, elle 
fait l'objet d'une coupe importante pour le bois de chauffe (Ouoba et al., 2006). Elle est généralement présente dans les savanes et les prairies boisées. C'est l'une des espèces caractéristiques des parties méridionales plus humides de la région soudanienne (Abonnier, 2009). Avec une distribution irrégulière, $M$. polyandra est fréquente dans les savanes guinéennes et soudanoguinéennes. Elle supporte les conditions édaphiques moyennes et est distribuée en Côte d'Ivoire, au Burkina Faso, au Cameroun et au Soudan (Sacande et al., 2012). Dans la Forêt classée de Niangoloko, M. polyandra colonise les sols ferrugineux tropicaux indurés profonds (plus de $60 \mathrm{~cm}$ ) (Ouoba, 2006).

Description de Maranthes polyandra (Benth.) Prance : La description de $M$. polyandra est celle rapportée par Arbonnier (2009) c'est-à-dire un petit arbre ou arbuste de 6-8 $\mathrm{m}$ de haut, bas branchu, à branches tortueuses et à cime ouverte (Figure 2A). Son écorce est crevassée, à écailles carrées noirâtres. Les rameaux sont pubescents avec des lenticelles, plus ou moins liégeux et de couleur brun orangé ou brun violacé. Les feuilles, de dimensions $6-13 \times 3-7,5 \mathrm{~cm}$, sont simples, alternes, coriaces, elliptiques ou obovales, à face supérieure glabre, vert foncé et à face inferieure pubescente, blanchâtre. Le sommet du limbe est arrondi très courtement et obtusément acuminé, à base arrondie ou en coin portant sur le dessus deux glandes circulaires de part et d'autre du pétiole. L'inflorescence de M. polyandra (Figure 2B) est une panicule de corymbes terminaux, tomenteuse, de $15-20 \mathrm{~cm}$ de long. De couleur blanche ou rose, la fleur est irrégulière, à 5 pétales, 5 sépales. Le fruit (Figure $2 \mathrm{C}$ ) est une drupe ovoïde ou ellipsoïde, glabre, rouge puis pourpre noirâtre à maturité, de 2 à $2,5 \mathrm{~cm}$ de long. La période de floraison de $M$. polyandra est très variable. Elle s'étend depuis la seconde moitié de la saison sèche (janvier-avril) jusqu'au milieu de la saison des pluies (juillet-août).

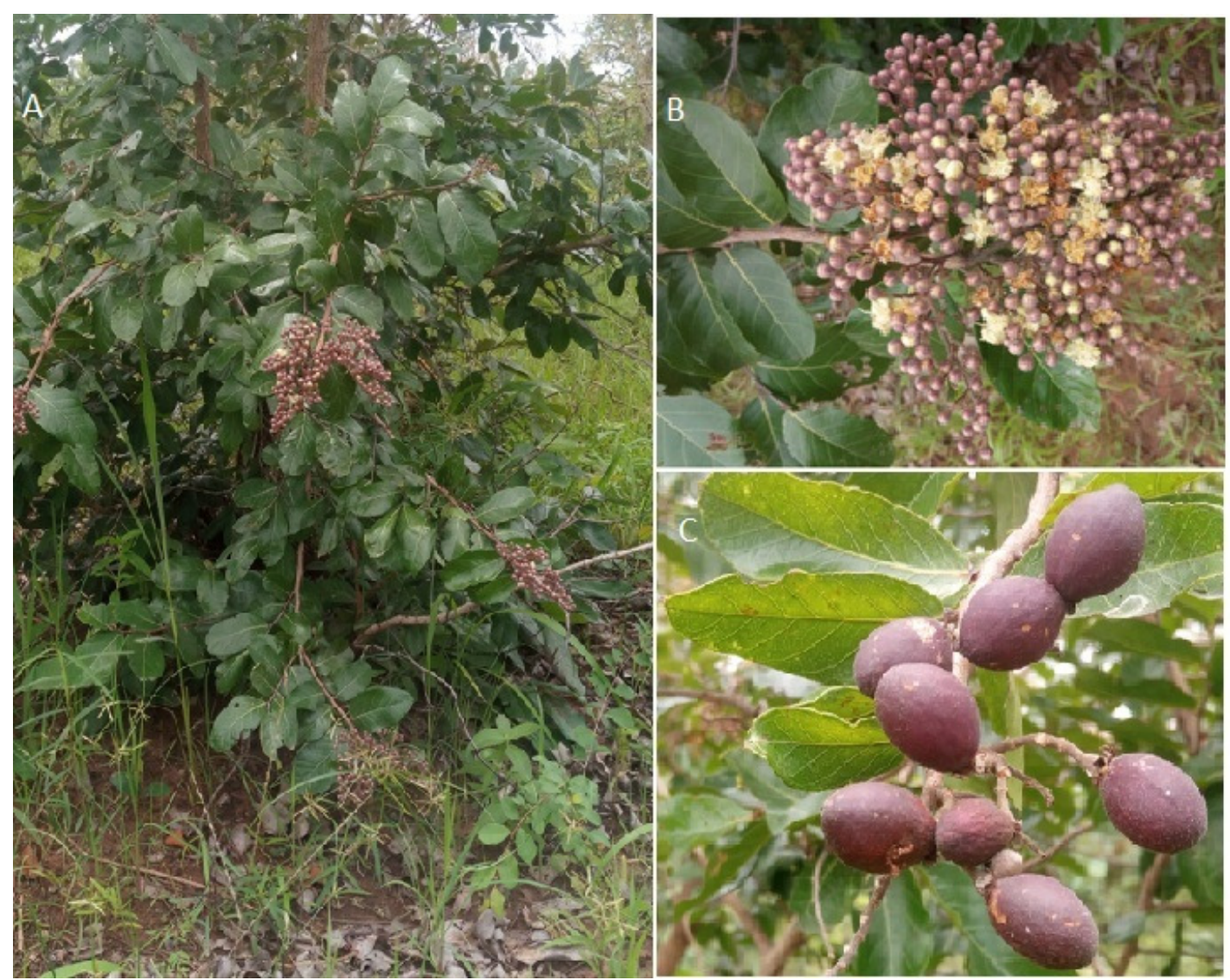

Figure 2 : Différentes parties de $M$. polyandra : le port (A), le rameau florifère $(B)$ et le rameau fructifère $(C)$.

Collecte de la litière foliaire et des échantillons de sol: Nous avons ramassé et pesé toute la litière des feuilles se trouvant sous le houppier de chaque pied de
M. polyandra et de Vitellaria paradoxa. L'étude physicochimique du sol sous le houppier et hors du houppier de M. polyandra et de V. paradoxa a consisté à prélever des 
échantillons. Au total, 30 individus de chaque espèce ont été considérés pour l'échantillonnage. A l'aide d'une tarière, les échantillons de sol ont été prélevés dans l'horizon 0-20 cm car selon Girard et al. (2011), la majorité de la biomasse aérienne tombée au sol est incorporée et concentrée dans cet horizon. Pour chaque arbre, les points de prélèvement ont été fixés suivant deux axes orientés est-ouest et nord-sud (Yélémou et al., 2013) de sorte à illustrer des situations sous houppier à la distance $r / 2$ ( $r$ étant le rayon du houppier) et hors houppier à la distance $2 x r$ soit 2 lots de 4 prélèvements. Pour chaque individu, un échantillon composite de sol sous houppier et un autre hors houppier ont été constitués pour les analyses physico-chimiques au laboratoire. Au total, 120 échantillons composites dont 60 pour M. polyandra et 60 pour $V$. Paradoxa ont été analysés.

Analyse des échantillons de sol et traitement des données : Les analyses au laboratoire ont porté sur la détermination de la granulométrie en trois fractions par la méthode du pipetage de Robinson-Koln, du pH eau (pH) par la méthode potentiométrique (AFNOR, 1994), du carbone $(\mathrm{C})$ organique par la méthode de Walkley-Black (1934), de l'azote total (N) par la méthode Kjeldahl (NKT)

\section{RÉSULTATS}

La litière foliaire: La production moyenne de litière foliaire de $M$. polyandra est significativement plus élevée que celle de $V$. paradoxa $(p=0,0001)$. La quantité moyenne de la litière produite par $M$. polyandra est de $28,82 \pm 16,08 \mathrm{~kg}$ par individus contre $5,92 \pm 3,07 \mathrm{~kg}$ pour $V$. paradoxa.

Caractéristiques physiques du sol: Le sol sous houppier de M. polyandra (MP_SH) a les plus fortes
(Bremner, 1965), du phosphore total (P_total) par la méthode Brayl (Reeuwijk, 2002), du potassium (K) total par la méthode de l'acétate d'ammonium, des cations échangeables et de la capacité d'échange cationique (CEC) par la méthode de l'acétate d'ammonium (Reeuwijk, 2002). La somme des bases échangeables (SBE) est alors obtenue par addition. La matière organique (MO) a été déduite du carbone par un facteur de conversion (1,72) (Mylavarapu, 2015). Les données sur les paramètres physico-chimiques du sol ont été soumises à une analyse de variance (ANOVA) avec le logiciel XLSTAT 7.5.2. Les moyennes des différentes variables ont été comparées à l'aide du test de Tukey au seuil de $5 \%$. Une Analyse en Composante Principale (ACP) a été faite avec le logiciel PC-ORD.6 (Mccune et Grace, 2002) afin d'établir les corrélations entre les paramètres chimiques et les zones de prélèvement (soushouppier et hors-houppier). Pour ce faire, la matrice des données avec en lignes les zones de prélèvement (sous houppier de M. polyandra (MP_SH), hors houppier de M. polyandra (MP_HP), sous houppier de V. paradoxa (VP_SH) et hors houppier de V. paradoxa (VP_HH)) et en colonnes les paramètres chimiques ( $\mathrm{pH}, \mathrm{C}, \mathrm{N}, \mathrm{P}, \mathrm{K}, \mathrm{SBE}$, $\mathrm{CEC}, \mathrm{MO}, \mathrm{C} / \mathrm{N}$ ) a été soumise à l'analyse.

teneurs en argile $\left(301 \mathrm{gkg}^{-1}\right)$ et en limon $\left(248 \mathrm{gkg}^{-1}\right)$ et, en parallèle la plus faible teneur en sable $\left(451 \mathrm{~g} \mathrm{~kg}^{-1}\right)$ (Tableau 1). L'analyse de variance a montré une différence significative à très hautement significative de ces trois paramètres entre les différentes zones de prélèvement (Tableau 1).

Tableau1 : Paramètres physiques du sol sous-houppiers et hors-houppiers des deux espèces

\begin{tabular}{l|l|l|l}
\hline Traitements & Argile $\left(\mathbf{g k g}^{-1}\right)$ & Limon $\left(\mathbf{g k g}^{-1}\right)$ & Sable $\left.\mathbf{~ g k g}^{-1}\right)$ \\
\hline MP_SH & $300,83^{\mathrm{a}} \pm 23,80$ & $248,37^{\mathrm{a}} \pm 19,97$ & $450,80^{\mathrm{b}} \pm 12,52$ \\
VP_SH & $261,67^{\mathrm{ab}} \pm 38,47$ & $233,23^{\mathrm{a}} \pm 10,16$ & $505,20^{\mathrm{a}} \pm 30,17$ \\
MP_HH & $243,78^{\mathrm{ab}} \pm 43,24$ & $217,92^{\mathrm{ab}} \pm 14,73$ & $538,30^{\mathrm{a}} \pm 36,51$ \\
VP_HH & $217,03^{\mathrm{b}} \pm 49,12$ & $206,70^{\mathrm{b}} \pm 33,55$ & $576,27^{\mathrm{a}} \pm 84,19$ \\
Probabilité & 0,01 & 0,02 & 0,001 \\
Signification & $\mathrm{S}$ & $\mathrm{S}$ & THS \\
\hline A & &
\end{tabular}

A l'intérieur d'une même colonne, les moyennes suivies de la même lettre ne sont pas significativement différentes au seuil de $5 \%$ selon le test de Tukey. S=Significatif ; THS= Très hautement significatif.- MP_SH : M. polyandra sous houppier; MP_HH : M. polyandra hors houppier ; VP_SH : V. paradoxa sous houppier; VP_HH : V. paradoxa hors houppier.

Caractéristiques chimiques du sol: Les caractéristiques chimiques des échantillons de sols analysés sont consignées dans le tableau 2. Le pH du sol ne varie pas significativement entre les deux espèces et les zones de prélèvement. Le sol est acide pour toutes les zones de prélèvement (moyenne 6,13). La zone soushouppier de $M$. polyandra a des teneurs en carbone organique très significativement plus élevées $(P<0,001)$ 
que les autres zones de prélèvement. Sa teneur en carbone est de $127 \%, 140 \%$ et $143 \%$ respectivement plus élevé que les zones VP_SH, MP_HH et VP_HH. La teneur en azote total a également été plus importante sous le houppier de M. polyandra par rapport aux autres zones de prélèvement $(p<0,001)$. Les valeurs du rapport $\mathrm{C} / \mathrm{N}$ voisines de 14 ne varient pas significativement entre les deux espèces et les différentes zones de prélèvement $(p>0,05)$. Pour le phosphore total, la variation est très significative $(p<0,001)$ entre les deux espèces et les zones de prélèvement du sol. La plus forte teneur a été observée sous le houppier de M. polyandra (Tableau 2). Pour ce qui concerne le potassium total, les variations sont aussi significatives $(P<0,05)$; le sol sous le houppier de $M$. polyandra a montré une teneur significativement plus élevée que les autres zones de prélèvement. L'analyse de variance a révélé une différence hautement significative $(p<0,001)$ pour les paramètres tels que la somme des bases échangeables (SBE) et la capacité d'échange cationique (CEC) entre les différentes zones de prélèvement. La SBE de $2,94 \mathrm{Cmol}^{+} . \mathrm{kg}^{-1}$ sol sous le houppier de $M$. polyandra est trois fois supérieure à celle de VP_SH, MP_HH et près de quatre fois supérieure à celle de VP_HH. Quant à la CEC avec 4,22 $\mathrm{Cmol}^{+} . \mathrm{kg}^{-1} \mathrm{sol}$, elle est trois fois supérieure sous $M$. polyandra comparée aux autres zones de prélèvement. Cependant, le taux de saturation de $70,90 \%$ sous $M$. polyandra n'a pas varié significativement $(p>0,05)$ entre les espèces et les différentes zones. 
Tableau 2: Valeurs des paramètres chimiques du sol en fonction des deux espèces et des zones de prélèvement

\begin{tabular}{|c|c|c|c|c|c|c|c|c|c|}
\hline $\begin{array}{l}\text { Zone de } \\
\text { prélèvement- }\end{array}$ & $\mathrm{pH}$ eau & C (\%) & N (\%) & $\mathrm{C} / \mathrm{N}$ & P-total $\left(\mathrm{mg} \mathrm{kg}^{-1}\right)$ & K-total(mg.kg-1) & $\begin{array}{l}\text { SBE } \\
\text { (Cmol } . \mathrm{kg}^{-1} \text { sol) }\end{array}$ & $\begin{array}{l}\text { CEC } \\
\text { (Cmol+..kg-1 sol) }\end{array}$ & V (\%) \\
\hline MP_SH & $6,20^{a} \pm 0,47$ & $1,68^{a} \pm 0,55$ & $0,12^{\mathrm{a}} \pm 0,04$ & $14,52^{\mathrm{a}} \pm 1,1$ & $96,25^{\mathrm{a}} \pm 36,07$ & $889,32^{a} \pm 456,01$ & $2,94^{\mathrm{a}} \pm 1,35$ & $4,22^{\mathrm{a}} \pm 1,94$ & $70,90^{a} \pm 13,55$ \\
\hline VP_SH & $6,16^{a} \pm 0,31$ & $0,74^{b} \pm 0,17$ & $0,05^{b} \pm 0,01$ & $14,10^{a} \pm 1,55$ & $60,68^{b} \pm 13,87$ & $734,40^{\mathrm{ab}} \pm 320,92$ & $1,08^{b} \pm 0,34$ & $1,61^{b} \pm 0,73$ & $70,34 a \pm 11,30$ \\
\hline MP_HH & $6,13^{a} \pm 0,35$ & $0,70^{b} \pm 0,29$ & $0,05^{b} \pm 0,02$ & $14,00^{a} \pm 1,82$ & $54,74^{\mathrm{b}} \pm 23,67$ & $695,92^{\mathrm{ab}} \pm 520,73$ & $0,88^{b} \pm 0,23$ & $1,33^{b} \pm 0,40$ & $68,33^{a} \pm 13,96$ \\
\hline VP_HH & $6,06 a \pm 0,24$ & $0,69^{b} \pm 0,18$ & $0,05^{b} \pm 0,01$ & $13,60^{a} \pm 1,5$ & $54,73^{b} \pm 21,55$ & $614,05^{b} \pm 228,51$ & $0,82^{b} \pm 0,31$ & $1,28^{b} \pm 0,72$ & $68,30^{a} \pm 14,33$ \\
\hline Probabilité/ $p$-value & 0,47 & $<0,001$ & $<0,001$ & 0,14 & $<0,001$ & 0,04 & 0,001 & 0,001 & 0,819 \\
\hline
\end{tabular}


L'analyse en composante principale (ACP) a montré que tous les paramètres chimiques sont fortement corrélés à la zone sous-houppier de M. polyandra (Figure 3). L'effet de M. polyandra est particulièrement marqué car le premier axe qui discrimine les différents espaces de prélèvement (MP_SH, VP_SH, MP_HH et VP_HH) explique plus de $92 \%(p<0,05)$ de la variance totale observée (Figure 3 et Tableau 3).

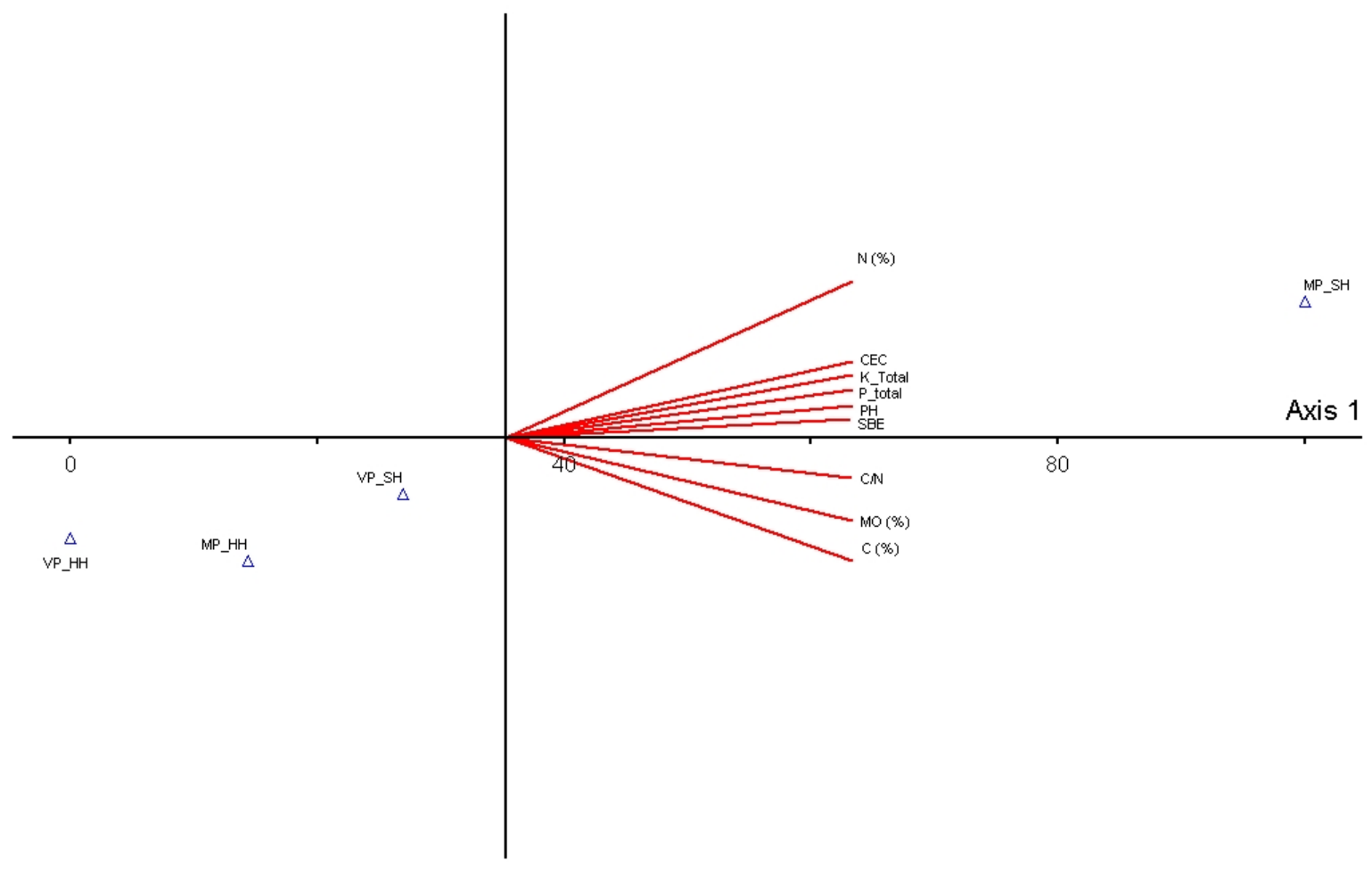

Figure 3. Plan factoriel de l'Analyse en Composante Principale

Tableau 3. Valeurs propres des 2 premiers axes

\begin{tabular}{lll}
\hline Axe & 1 & 2 \\
\hline Valeur propre & 8,34 & 0,61 \\
Pourcentage de la variance & 92,65 & 6,80 \\
Probabilité & 0,001 & 1,00 \\
\hline
\end{tabular}

\section{DISCUSSION}

Nous avons trouvé que $M$. polyandra a produit plus de litière de feuilles que $V$. paradoxa. Les performances variables de production de litière entre les ligneux ont été rapportées par d'autres études. Ainsi, Gnahoua et al. (2013) ont montré que Leucaena leucocephala (Lam.) De Wit avait une production annuelle de litière foliaire plus élevée que trois autres espèces végétales que sont Acacia auriculiformis A. Cunn. Ex Benth., Acacia mangium Willd. Albizia lebbeck (L) Benth. Le dépôt important de litière sous $M$. polyandra peut expliquer les valeurs élevées des paramètres de fertilité du sol sous son houppier comparativement au sol sous le houppier de V. paradoxa. Le houppier de M. polyandra a contribué de manière significative à améliorer la texture du sol. Les plus fortes teneurs d'argile $\left(301 \mathrm{gkg}^{-1}\right)$ et de limons (248 $\mathrm{gkg}^{-1}$ ) y ont été enregistrées. Les valeurs de ces paramètres (argile, limon et sable par différence) traduisent l'impact du houppier dans l'atténuation de l'érosion que pourraient engendrer l'eau de pluie et aussi le rôle joué par l'important dépôt de la litière. En effet, la litière protège certainement le sol sous le houppier de $M$. polyandra de l'énergie cinétique de la pluie, ce qui limite le ruissellement et le départ des éléments fins de la surface du sol, au contraire de la situation hors du houppier. Les mêmes constats ont été faits par Yélémou et al. (2013) sur Piliostigma thonningii (Schumach.) Milne- 
Redh.et $P$. reticulatum (DC.) Hochst ainsi que par Traoré et al. (2007) sur Acacia spp. En outre, l'important dépôt de litière sous le houppier de $M$. polyandra favorise certainement l'activité de la macrofaune du sol (vers de terre, termites, etc.) (Criquet et al., 2002 ; Andersson et al., 2004) et, par conséquent, la remontée dans l'horizon de surface d'éléments fins provenant des horizons de profondeur. Cette activité de la microfaune va donc modifier la texture du sol sous $M$. polyandra en le rendant plus argileux, au contraire de la situation hors houppier. Ce remaniement de la texture du sol sous $M$. polyandra peut avoir des conséquences sur ses propriétés chimiques. En effet, plusieurs travaux ont montré que la texture a un effet sur le stockage et la dynamique de plusieurs paramètres chimiques au niveau des horizons de surface (Feller et al. 1991; Zinn et al., 2005). Par exemple, Feller et al. (1991) ont montré qu'il existait une relation étroite entre la teneur en carbone organique et la teneur en argile des sols. Ces résultats vont dans le même sens que ceux que nous avons trouvé dans la présente étude en ce sens que la teneur en carbone organique et la teneur en argile des sols sont plus élevées sous le houppier de $M$. polyandra. Le sol sous le houppier de $M$. polyandra se distingue ainsi par des teneurs élevées en matière organique $(2,9 \%)$. Selon Barton et al. (2016), l'accroissement de la matière organique a de multiples bénéfices pour les sols agricoles dans les climats semi-arides. II améliore la résilience de la texture du sol en réduisant le ruissellement et en augmentant la capacité de rétention des nutriments et de l'infiltration (Hoyle et al.,2011). D'autres auteurs (Li et al., 2013 ; Nave et al., 2010) trouvent que la grande quantité de matière organique de la litière des feuilles affectent positivement la matière organique du sol et est considérée comme un bon indicateur pour le ravitaillement en nutriments du sol, de l'amélioration de ses propriétés chimiques et de la prévention de l'érosion. Cela est davantage illustré par la valeur plus élevée du rapport $\mathrm{C} / \mathrm{N}$ du sol sous le houppier de M. Polyandra qui traduit une minéralisation quelque peu ralentie de la matière organique et par conséquent, la séquestration du carbone dans le sol (Fontaine et al., 2004 ; Pallo et al., 2009 ; Alberti et al., 2015). Aussi, Tanaka et Hashimoto (2006), Jandl et al. (2007) rapportent que les ligneux contribuent considérablement à la séquestration du carbone dans les régions arides et semi-arides. D'une manière générale, les plus fortes teneurs en éléments majeurs tels que l'azote total $(0,12 \%)$, le phosphore total (96,25 mg.kg-1) et le potassium total $(889,32 \mathrm{mg} . \mathrm{kg}-1)$ ainsi que les plus fortes valeurs en SBE $(2,94 \mathrm{Cmol}+\mathrm{kg}$ 1sol) et en CEC (4,22 Cmol+kg-1sol) sont enregistrées sous M. polyandra. Ces fortes valeurs sont probablement liées à la décomposition de la masse importante de litière que produisent les individus de cette espèce mais pourraient aussi provenir de la différence de texture observée entre les sols sous houppier et hors houppier de M. polyandra. En effet, les arbres ont le potentiel d'influencer quantitativement et qualitativement les propriétés du sol à travers la litière de leurs feuilles et leurs racines (Kamei et al., 2009 ; Talkner et al., 2009; Wang et al., 2011). Alig (2003) et Alarcón-Gutiérrez (2007) soutiennent que la décomposition des litières forestières permet le recyclage de nombreux éléments chimiques tels que le carbone, l'azote, le phosphore et le soufre. La forte teneur en azote total sous le houppier de M. polyandra pourrait être liée à l'abondante litière foliaire de l'espèce comme l'a rapporté Yaméogo et al. (2005) pour d'autres espèces ligneuses. La forte teneur en phosphore sous $M$. polyandra serait une conséquence de la matière organique du sol car cette dernière contient 20 à $80 \%$ du phosphore des sols (Winterbottom et al., 2013), mais pourrait être aussi expliquer par les dépôts atmosphériques (captés par le houppier de M. polyandra) ou la remontée du phosphore des horizons profonds vers les horizons superficiels grâce aux racines profondes de $M$. polyandra. Les résultats indiquent qu'il n'y a pas de différence significative entre la zone sous-houppier et hors-houppier de $\mathrm{V}$. paradoxa pour le $\mathrm{pH}$, la matière organique, le rapport $\mathrm{C} / \mathrm{N}$, le carbone, l'azote, le phosphore et le potassium. Nos résultats corroborent ceux de Diarra (2009) qui n'a pas observé de différence entre le $\mathrm{pH}$, la teneur en matière organique et en phosphore, sous et hors houppier de V. paradoxa. En outre, Saidou et al. (2012) ont abouti aux mêmes résultats en trouvant qu'il n'y a pas de différence significative dans la teneur des éléments chimiques du sol entre l'espace sous houppier et hors houppier de $V$. paradoxa à l'exception du $\mathrm{C}$ et $\mathrm{N}$ qui sont légèrement plus élevés sous son houppier. Ces résultats montrent que l'impact de la présence de $V$. paradoxa est relativement faible sur les paramètres physico-chimiques du sol bien qu'il constitue l'un des principaux arbres conservés dans les parcs agroforestiers. Maranthes polyandra, apparaitt donc être potentiellement une plante agroforestière qui pourraient être valorisée. Baumer (1987) rapporte qu'au Burkina Faso, plus de la moitié des terres sont pauvres en matière organique, $85 \%$ sont très pauvres en phosphore et $61 \%$ sont très pauvres en azote. Pierce et Lal (1994) et Zougmoré et al. (2009) indiquaient que le niveau bas en N, P, K et de la capacité d'échange cationique constituait la contrainte majeure pour la production agricole. 


\section{CONCLUSION}

Ce travail à permis d'étudier les relations entre $M$. polyandra et les propriétés physiques et chimiques du sol. II a montré que l'espèce a un potentiel à améliorer la fertilité physique et chimique du sol. En comparant les résultats obtenus sous M.polyandra à ceux sous $V$. paradoxa, on note que $M$. polyandra produit plus de litière et contribue significativement à l'amélioration de la texture du sol d'une part et d'autre part à l'augmentation de la teneur du sol en carbone, azote, phosphore, potassium,

\section{BIBLIOGRAPHIE}

AFNOR., 1994. Détermination du pH. NF ISO 10390. AFNOR-qualité des sols, Paris.

Alarcón-Gutierrez E., 2007. Influence de facteurs abiotiques sur la régulation des paramètres microbiens impliqués dans la dégradation de la matière organique d'une litière forestière méditerranéenne. Thèse de docteur : Université Paul Cezanne-Faculté des sciences et techniques, Marseille (France).

Alberti G., Vicca S., Inglima I., Belelli-Marchesini L., Genesio L., Miglietta F., Marjanovic H., Martinez C., Matteucci G., D'Andrea E., Peressotti A., Petrella F., Rodeghiero M. \& Cotrufo M.F., 2015. Soil C:N stoichiometry controls carbon sink partitioning between above-ground tree biomass and soil organic matter in high fertility forests. iForest - Biogeosciences and Forestry, 8, 195206.

Alig R.J., 2003. U.S. landowner behavior, land use and land cover changes, and climate change mitigation. Silva Fennica, 37(4), 511-527.

Andersson M., Kjøller A. \&Struwe S., 2004. Microbial enzyme activities in leaf litter, humus and mineral soil layers of European forests. Soil Biology \& Biochemistry, 36, 1527-1537.

Arbonnier M., 2009. Arbres, arbustes et lianes des zones sèches d'Afrique de l'Ouest. Versailles, France : Quae, $573 \mathrm{p}$.

Barton L., Hoyle F. C., Stefanova K. T., \&Murphy D. V., 2016. Incorporating organic matter alters soil greenhouse gas emissions and increases grain yield in a semi-arid climate. Agriculture, Ecosystems and Environment, 231, 320-330.

Bationo A., Kalinganiré A. \& Bayala J., 2012. Potentialités des ligneux dans la pratique de l'agriculture de conservation dans les zones arides et semiarides de l'Afrique de l'Ouest: Aperçu de quelques systèmes candidats. Technical Manual no. 17 : ICRAF-World Agroforestry Center, somme des bases échangeables et capacité d'échange cationique. L'espèce a un fort potentiel agroforestier et pourrait être valorisée dans les systèmes à parcs agroforestiers pour promouvoir une agriculture durable. Ce qui peut constituer un motif supplémentaire pour sa conservation. Néanmoins d'autres études sur la composition chimique de la litière de ses feuilles et la macrofaune du sol sous son houppier pourront permettre de mieux apprécier son potentiel.

Nairobi (Kenya).

Bationo A. \& Buerkert, A. 2001. Soil organic carbon management for sustainable land use in Sudano-Sahelian West Africa. Nutr. Cycl. Agroecosyst., 61, 131-142.

Bationo A., 1994. Étude des potentialités agroforestières, de la multiplication et des usages de Guiera senegalensis J.F.GMEL. Mémoire de DEA: Université de Ouagadougou-Faculté des sciences et techniques, Ouagadougou (Burkina Faso).

Baumer M., 1987. Agroforesterie et Désertification. Wageningen, CTA, Pays-Bas, ICRAF, CTA. ,

Bayala J., Balesdent J., Marol C., Zapata F., Teklehaimanot Z. \& Ouedraogo S.J., 2006. Relative contribution of trees and crops to soil carbon content in a parkland system in Burkina Faso using variations in natural ${ }^{13} \mathrm{C}$ abundance. Nutr.Cycl.Agroecosys., 76, 193-201.

Bayala J., Mando A., Ouedraogo S.J. \&Teklehaimanot Z., 2003. Managing Parkia biglobosa and Vitellaria paradoxa Prunings for Crop Production and Improved Soil Properties in the Sub-Sudanian Zone of Burkina Faso. Arid Land Research and Management, 17(3), 283-296.

Bremner J.M., 1965. Inorganic Forms of Nitrogen, Methods of Soils Analysis. Am. Soc. Agron 1179 $-1237$.

BUNASOL, 1996. Étude morpho-pédologique des Forêts Classées du Houet et de la Comoé. Projet Carto, Ouagadougou, $65 \mathrm{p}$.

Coulibaly K., Gomgnimbou A.P.K., Bacye B., Nacro H.B. \& Sedogo M.P., 2016. Valorization of shea caterpillar droppings (Cirina butyrospermi Vuillet) in the ecological management of soil fertility in Burkina Faso. Int. J. Agron. Agri. Res., 9(1), 1-8. Coulibaly K., Vall E., Autfray P., Nacro H.B \& Sedogo P.M., 2012. Effets de la culture permanente 
coton-maïs sur l'évolution d'indicateurs de fertilité des sols de l'Ouest du Burkina Faso. Int. J. Biol. Chem. Sci., 6(3), 1069-1080.

Criquet S., Tagger S., Vogt G. \& Le Petit J., 2002. Endoglucanase and B-glycosidase activities in an evergreen oak litter: annual variation and regulating factors. Soil Biology \& Biochemistry, 34, 1111-1120.

CTFT, 1989. Faidherbia albida (Del.) A. Chev. (Synonyme : Acacia albida Del..). Caractères sylvicoles et méthodes de plantation. Bois et Forêts des Tropiques, 222, 55-68.

Diarra B. G., 2009. Influence du phosphore, de l'azote et $d u$ houppier sur les rendements du sorgho (Sorghum bicolor), les fractions du Phosphore et l'activité des microorganismes du sol d'un parc agroforestier de la zone soudanienne du Burkina Faso. Mémoire d'ingénieur agronome: Université polytechnique de Bobo-Dioulasso, Institut du Développement rural, Bobo-Dioulasso (Burkina Faso).

Feller C., Fritsch E., Poss R. Valentin C., 1991. Effet de la texture sur le stockage et la dynamique des matières organiques dans quelques sols ferrugineux et ferralitiques (Afrique de l'ouest, en particulier). Cahiers de I'ORSTOM, série Pédologie, 26 : 25-36.

Fontaine S., Bardoux G., Abbadie L. \& Mariotti A., 2004. Carbon input to soil may decrease soil carbon content. Ecology Letters, 7, 314-320. -doi: 10.1111/j.1461-0248.2004.00579.x

Fontes J. \& Guinko S., 1995. Carte de la végétation et de l'occupation du sol du Burkina Faso. Note explicative. Toulouse, France, Ministère de la coopération française, $65 p$.

Girard M-C., Walter C., Rémy J-C., Berthelin J. \& Morel J-L., 2011. Sols et environnement. 2è éd. Paris, DUNOD.

Gnahoua G. M., Robert O., Nguessan K. A. \& Balle P., 2013. Production et retombées minérales des litières chez des légumineuses arborées, utilisées en amélioration des jachères. J. Appl. Biosci., 72, 5800- 5809.

Hoyle F.C., Baldock J.A. \& Murphy D.V., 2011. Soil organic carbon role in rainfed farming systems with particular reference to Australian conditions. In: Tow P., Cooper I., Partridge I., Birch C. Eds., Rainfed Farming Systems. Springer International, 339-361. https://link.springer.com/chapter/10.1007\%2F97 8-1-4020-9132-2 14\#page-1 (Consulté le
20/12/2016).

Jandl R, Lindner M ., Vesterdal L., Bauwens B., Baritz R., Hagedorn F., Johnson D. W., Minkkinen K. \& Byrne K.A., 2007. How strongly can forest management influence soil carbon sequestration? Geoderma, 137, 253-268.

Kaboré S.A., Bastide B., Traoré S. \& Boussim J.I., 2012. Dynamique du karité, Vitellaria paradoxa, dans les systèmes agraires du Burkina Faso. Bois et forêts des tropiques, 313 (3), 47-59.

Kamei J., Pandey H.N.\& Barik S.K., 2009. Tree species distribution and its impact on soil properties, and nitrogen and phosphorus mineralization in a humid subtropical forest ecosystem of northeastern India. Can. J. For. Res., 39, 36-47.

Li Y., Brandle J. R., Awada T., Chen Y., Han J., Zhang F. \& Luo Y., 2013. Accumulation of carbon and nitrogen in the plant-soil system after afforestation of active sand dunes in China's Horqin Sandy Land. Agriculture, Ecosystems \& Environment, 177: 75-84.

Makumba W., Akinnifesi F.K., Janssen B. \&Oenema O., 2007. Long-term impact of a gliricidia-maize intercropping system on carbon sequestration in southern Malawi, Agriculture, Ecosystems and Environment, 118, 237-243.

Mccune B. \& Grace, J.B. 2002. Analysis of Ecological communities.Gleneden/Beach, USA, MjM software Design.

Mylavarapu R., 2015. Walkley-Black Method. www.clemson.edu/sera6/WalkleyBlackFinal0527 11.doc

Nave L.E, Vance E.D, Swanston C.W. and Curtis P.S., 2010. Harvest impacts on soil carbon storage in temperate forests. Forest Ecology and Management, 259(5): 857-866.

Ouoba P., 2006. Flore et végétation de la forêt classée de Niangoloko, sud-ouest du Burkina Faso. Thèse de doctorat : Université de Ouagadougou, UFR-SVT, Ouagadougou.

Ouoba P., Boussim I. \& Guinko S., 2006. Le potentiel fruitier de la Forêt Classée de Niangoloko. Fruits, 61 (1),71-81.

Pallo F.J.P., Sawadogo N., Zombré N.P. \& Sedogo M.P., 2009. Statut de la matière organique des sols de la zone nord-soudanienne au Burkina Faso. Biotechnol. Agron. Soc. Environ., 13(1), 139142.

Pierce F. J. \& Lal R., 1994. Monitoring soil erosion's impact on crop productivity, in R. Lal, ed., Soil erosion research methods, Ankeny, USA : Soil 
and water conservation society, 235-263.

Reeuwijk L.P. van, 2002. Procedures for soil analysis. $6^{e}$ ed. , Wageningen, Netherlands; ISRIC-Word Soil Information.https://www.google.fr/?gws rd=ssl\#q=Reeuwijk, +L.P. + van, $+2002 .+$ Procedur es+for+soil+analysis.+6e+ed.,++ Wageningen,+ Netherlands; +ISRIC-Word+Soil+Information. (Consulté le 12/4/2016)

Roupsard O., 1997. Ecophysiologie et Diversité Génétique de Faidherbia albida (Del.) A. Chev. (Syn. Acacia albida Del.), un Arbre à Usage Multiple d'Afrique Sémi-Aride. Thèse de doctorat, Université H. Poincaré de Nancy 1 , France.

Sacande M, Sanou L. \& Beentje H., 2012. Guide d'i'dentification des arbres du Burkina Faso. Kew, Royaume-Uni, $288 \mathrm{p}$.

Saidou A., Bologoun I., Koné B., Gnangle C.P. \& Aho N., 2012. Effet d'un système agroforestier à karité (Vitellaria paradoxa c.f. gaertn ) sur le sol et le potentiel de production du maïs (Zea mays L.) en zone Soudanienne du Bénin. Int. J. Biol. Chem. Sci., 6(5), 2066-2082.

Scherr S.J. \& Sthapit S., 2009.Mitigating climate change through food and land use, World watch report 179, Washington, D.C. ISBN 978-1-878071-910 ,

p.http://www.worldwatch.org/system/files/179\%2 OLand\%20Use.pdf (Consulté le 20/10/2016)

Talkner U., Jansen M. \& Beese FO., 2009. Soil phosphorus status and turnover in centralEuropean beech forest ecosystems with differing tree species diversity. European Journal of Soil Science, 60,338-346.

Tanaka K. \& Hashimoto S., 2006. Plant canopy effects on soil thermal and hydrological properties and soil respiration. Ecological Modelling, 196(1-2), 32-44.

Traoré O., Koulibaly B.\& Dakuo D., 2007. Effets comparés de deux formes d'engrais sur les rendements et la nutrition minérale en zone cotonnière au Burkina Faso. Tropicultura, 25(4), 200-203.

Traoré S., Thiombiano L., Millogo J.R. et Guinko S., 2007. Carbon and nitrogen enhancement in Cambisols and Vertisols by Acacia spp. in eastern Burkina Faso: Relation to soil respiration and microbial biomass. Applied Soil Ecology, 35 (3), 660-669.
Walkley A. \& Black I. A., 1934. An examination method of the detjareff and a proposed modification of the chromic acid titration method. Soil science, 37 , 29-38.

Wang Q., Wang S. \& Yu X., 2011. Decline of soil fertility during forest conversion of secondary forest to Chinese fir plantation in subtropical China. Land Degrad. Develop., 22, 444-452.

Weigel J., 1994. Agroforesterie pratique. Paris, Ministère de la Coopération.

White F., 1983. The vegetation of Africa. A descriptive memoir to accompany the UNESCO/AETFAT/UNSO vegetation map of Africa. Paris, UNESCO.

Winterbottom R., Reij C., Garrity D., GloverJ., Hellums D., McGahuey M., et Scherr S., 2013. "Improving Land and Water Management". Washington, World Resources Institute Working Paper.

https://www.wri.org/sites/default/files/improving__ and and water management 0.pdf (Consulté le 18/9/2016)

Yaméogo G., Yélémou B. \&Traoré D., 2005.Pratique et perception paysannes dans la création de parc agroforestier dans le terroir de Vipalogo (Burkina Faso). Biotechnol. Agron. Soc., Environ., 9(4), 141-148.

Yaméogo T. J., Lykke A. M., Hien M. et Nacro H.B., 2013. Restauration des potentialités de sols dégradés à l'aide du zaï et des cordons pierreux à l'Ouest du Burkina Faso. Tropicultura, 31(4), 224-230.

Yélémou B., Dayamba S.D., Yaméogo D.B.G., Assimi S., 2013. Soil carbon and nitrogen dynamics linked to Piliostigma species in ferugino-tropical soils in the Sudano-Sahelian zone of Burkina Faso, West Africa. Journal of Forestry Research, 24(1),99-108.

Zinn Y.L., Lal R. et Resck V.S.D., 2005. Texture and organic carbon relations described by a profile pedotransfer function for Brazilian Cerrado soils. Geoderma, 127, 168-173.

Zougmoré R., Mando A. \& Stroosnijder L., 2009. Soil Nutrient and Sediment Loss as Affected By Erosion Barriers and Nutrient Source in SemiArid Burkina Faso'. Arid Land Research and Management, 23(1), 85 - 101. 